\title{
PRODUCCIÓN DE CAFÉ EN RESPUESTA AL FRACCIONAMIENTO DE LA FERTILIZACIÓN
}

\author{
Hernán González Osorio (D) *, Siavosh Sadeghian Khalajabadi (D) **
}

\begin{abstract}
González-Osorio, H., \& Sadeghian, S. (2020). Producción de café en respuesta al fraccionamiento de la fertilización. Revista Cenicafé, 71(1), 54-67. https://doi.org/10.38141/10778/1120
\end{abstract}

La fertilización constituye una práctica clave en la producción de café y su efectividad depende de la pertinencia y oportunidad con la que se efectúe. Considerando la diversidad de suelos en los que se cultiva el café en Colombia, el costo de los fertilizantes y las variaciones de los factores climáticos que inciden en el cultivo, el fraccionamiento de la fertilización es una estrategia tendiente al incremento de la productividad. El presente estudio tuvo como objetivo evaluar el efecto del fraccionamiento de la fertilización en la producción anual de café y en el acumulado del ciclo productivo, en seis localidades de la zona cafetera (El Agrado-Quindío, El Rosario-Antioquia, Naranjal-Caldas, Paraguaicito-Quindío, El Porvenir-Caldas y San Antonio-Santander), con características climáticas y de suelos diferentes. Las dosis de fertilizante se establecieron según las recomendaciones del análisis de suelo, para cada localidad y se aplicó fraccionado en dos, tres, cuatro y seis veces al año. En cinco localidades no hubo efecto del fraccionamiento, pese a que la pluviosidad superó en al menos dos años de evaluación, los niveles históricos de más de 30 años. En Paraguaicito, cuyo suelo presenta alta conductividad hidráulica, fraccionar el fertilizante en cuatro y seis oportunidades condujo al aumento promedio del $12 \%$ en la producción solamente en uno de los cuatro años evaluados. Los resultados permiten concluir que el suministro del fertilizante requerido según el análisis de suelos puede hacerse en dos fracciones por año.

Palabras clave: Conductividad hidráulica, efectividad de la fertilización, ENOS- La Niña, textura, zona cafetera colombiana.

\section{COFFEE YIELD RESPONSE TO SPLIT FERTILIZATION}

Fertilization is a key practice in coffee production, its effectivity depends on relevance and timing. Considering the diversity of soils used to grow coffee in Colombia, the cost of fertilizers and the variations in climatic factors that affect cultivation, split fertilization is a strategy aimed at increasing productivity. The main objective of our study was to evaluate the effect of split fertilization on annual coffee production as the accumulated of the productive cycle in six localities of the coffee zone (El Agrado-Quindío, El RosarioAntioquia, Naranjal-Caldas, Paraguaicito-Quindío, El Porvenir-Caldas and San Antonio-Santander) that have different climatic and soil characteristics. Fertilizers doses were established according to the recommendations of the soil analysis for each locality and applied split into two, three, four, and six times per year. There was no split effect in five localities, despite the fact that the rainfall exceeded the historical levels of more than 30 years in at least two years of evaluation. At Paraguaicito, whose soil has high hydraulic conductivity, the split fertilization at four and six times per year increased around $12 \%$ in production only in one of the four years evaluated. The results show that the supply of the required fertilizer, according to the soil analysis, can be done in two splits per year.

Keywords: hydraulic conductivity, fertilization effectiveness, ENSO- La Niña, soil texture, Colombian coffee region.

\footnotetext{
* Investigador Científico I. Disciplina de Suelos, Centro Nacional de Investigaciones de Café, Cenicafé. Chinchiná, Caldas, Colombia. https://orcid.org/0000-0001-5716-2172.

** Investigador Científico III. Disciplina de Suelos, Centro Nacional de Investigaciones de Café, Cenicafé. Chinchiná, Caldas, Colombia. https://orcid.org/0000-0003-1266-0885.
} 
El crecimiento, desarrollo y producción de los cultivos se deriva entre otros aspectos, de un programa adecuado y oportuno de fertilización (McArthur y McCord, 2019; Ierna y Mauromicale, 2018). Por medio de esta práctica se busca corregir y prevenir las deficiencias nutricionales de la plantación, contribuir con la calidad de las cosechas y sostener la fertilidad del suelo con el fin de que las plantas puedan nutrirse en el momento que lo necesiten. La eficiencia en esta labor depende de la oportunidad con la que se realice, de las características del suelo y las condiciones climáticas incidentes en los sistemas de producción principalmente (Arcila, 2007).

La selección de las fuentes fertilizantes puede incidir en el aprovechamiento de los nutrientes aplicados en los planes de fertilización. Algunas incluyen en su formulación moléculas que contribuyen a reducir las pérdidas de nutrientes por volatilización (Afshar et al., 2018), son de liberación gradual (Niemiec et al., 2015) o contienen formas químicas predominantes que al ser adsorbidas en el suelo retardan la lixiviación (Tian et al., 2018; Cao et al., 2014). Pese a que estas alternativas se vienen desarrollando con miras al incremento de la productividad y la reducción de riesgos ambientales en diferentes regiones del mundo (Zhang et al., 2018), su utilización no es garantía de que la nutrición de las plantas sea eficiente; se requiere entonces tanto para este tipo de fertilizantes como para las fuentes convencionales, que en su implementación se integren otros factores que inciden en los sistemas de producción (Liu et al., 2017; Tully et al., 2012), particularmente en áreas diversas en recursos naturales como la zona cafetera de Colombia. Por lo mencionado, el ajuste a los factores que determinan la eficiencia a la fertilización ha sido labor permanente para la caficultura del país como dosis de fertilizantes (Sadeghian y González, 2012), fuentes (Sadeghian et al., 2007), formas de aplicación y su fraccionamiento a través del tiempo (Mestre y Uribe, 1980).

En café, con base en estos y otros criterios, desde la década del 70 se planteó la necesidad de definir la frecuencia de aplicación de fertilizantes para diferentes regiones de la zona cafetera del país y fue a partir de estos estudios, que se generó la recomendación de suministrar el fertilizante requerido por año para cafetales en producción cada seis meses, basados en el hecho de que mediante el empleo de dicha práctica se obtienen los mayores beneficios desde el punto de vista económico (Mestre y Uribe, 1980). Sin embargo, aún persisten interrogantes si se debe o no fraccionar, en más de dos oportunidades la fertilización puesto que, una vez se disuelven los fertilizantes, los nutrientes en ellos contenidos quedan a disposición de las raíces del cultivo, en caso tal que la planta pueda absorberlos, una aparte queda como reserva en el suelo (incluida la inmovilización microbiana) y otro tanto en el agua que se percola en el suelo y los conduce a sitios donde la raíz no alcanza (Ghiberto et al., 2009; Sadeghian et al., 2015).

Por lo mencionado, ante eventos de variabilidad climática, la generación de nuevas variedades con atributos de producción y calidad, así como la volatilidad en el precio de los fertilizantes, se hace necesario continuar en la búsqueda de alternativas que contribuyan con el aumento de la productividad en la zona cafetera colombiana. Por esta razón, se planteó como objetivo evaluar el efecto del fraccionamiento del fertilizante requerido por año, según el análisis de suelos en la producción del café, para diferentes condiciones edáficas y ambientales de la zona cafetera colombiana, como una estrategia de orientación para que la práctica de fertilización se refleje exitosamente desde el punto de vista productivo, económico y ambiental. 


\section{MATERIALES Y MÉTODOS}

Para alcanzar el objetivo, el presente experimento se desarrolló en plantaciones de café Variedad Castillo ${ }^{\circledR}$, ubicadas en seis localidades de la zona cafetera, con características de suelos y condiciones de pluviosidad diferentes (Tablas 1 y 2).

Durante la fase de crecimiento vegetativo, los cafetales recibieron una fertilización general basada en las recomendaciones que para tal caso tiene Cenicafé (Sadeghian, 2008), de acuerdo con los resultados del análisis de suelos (Tabla 2).

Una vez la plantación alcanzó 18 meses de edad, fue tomada una nueva muestra para su análisis, con el fin de establecer la fertilización de la fase reproductiva, en la cual se aplicaron los siguientes tratamientos, consistentes en el suministro de la fertilización requerida por año en cuatro diferentes épocas:

Tratamiento 1. Aplicación del fertilizante requerido fraccionado o distribuido en dos épocas del año $(50 \%$ en el primer semestre y $50 \%$ en el segundo), es decir, cada semestre.
Tratamiento 2. Aplicación del fertilizante distribuido en tres épocas en el año (33\% cada cuatro meses).

Tratamiento 3. Aplicación del fertilizante distribuido en cuatro épocas en el año (25\% cada tres meses).

Tratamiento 4. Aplicación del fertilizante distribuido en seis épocas del año, lo cual significa, que la fertilización se realizó cada dos meses (16\% cada vez).

En adelante y cada dos años se tomaron muestras de suelos en las parcelas correspondientes al tratamiento 1 , para hacer los ajustes en materia de fertilizantes y/o las enmiendas en la totalidad de los tratamientos evaluados.

Los meses seleccionados para la aplicación de los fertilizantes se mantuvieron durante los cuatro años de producción (Tabla 3), considerando necesario que existiera humedad suficiente en el suelo para efectuar la labor. Cuando por carencia de lluvias no hubiese sido posible realizarla, se dio espera hasta tanto el evento de lluvia se hiciera manifiesto.

Tabla 1. Ubicación y características de los sitios experimentales.

\begin{tabular}{ccccccc}
\hline $\begin{array}{c}\text { Departamento } \\
\text { Municipio }\end{array}$ & Quindío & Antioquia & Caldas & Quindío & Caldas & Santander \\
Finca & El Agrado & El Rosario & Naranjal & Paraguaicito & El Porvenir & San Antonio \\
Altitud (m) & 1.250 & 1.600 & 1.400 & 1.250 & 1.400 & 1.480 \\
$\begin{array}{c}\text { Lluvia (mm año } \\
\text { Plantas por }\end{array}$ & 1.810 & 2.230 & 2.400 & 2.100 & 4.013 & 1.160 \\
$\begin{array}{c}\text { hectárea } \\
\text { Inicio del }\end{array}$ & 10.000 & 5.917 & 8.888 & 5.917 & 5.917 & 5.917 \\
experimento (año) & 2010 & 2010 & 2009 & 2011 & 2011 & 2010 \\
\hline
\end{tabular}


Tabla 2. Características de los suelos antes de la aplicación de los tratamientos

\begin{tabular}{|c|c|c|c|c|c|c|c|}
\hline & & El Agrado & El Rosario & Naranjal & Paraguaicito & El Porvenir & San Antonio \\
\hline $\begin{array}{l}\text { Materia } \\
\text { Orgánica }\end{array}$ & $\%$ & 5,8 & 9,3 & 14,3 & 6,2 & 12,8 & 5,6 \\
\hline pH & & 4,4 & 4,5 & 5,3 & 5,1 & 5,0 & 5,0 \\
\hline $\mathbf{P}$ & $\mathrm{mg} \mathrm{kg}^{-1}$ & 210,0 & 29,8 & 34,0 & 115,0 & 5,0 & 12,0 \\
\hline $\mathbf{K}$ & & 0,3 & 0,7 & 0,3 & 0,2 & 0,4 & 0,2 \\
\hline $\mathbf{C a}$ & $\mathbf{c m o l}_{\mathrm{c}}$ & 1,0 & 2,4 & 3,24 & 3,3 & 2,1 & 2,9 \\
\hline Mg & $\mathbf{k g}^{-1}$ & 0,1 & 0,6 & 1,2 & 0,4 & 0,5 & 0,6 \\
\hline Al & & 2,2 & 6,1 & 0,8 & 0,8 & 1,0 & 0,9 \\
\hline Textura & Clase & F.Ar.A.* & F.Ar.A. & F.A.** & F.Ar.A. & F.A. & F.Ar.A. \\
\hline
\end{tabular}

*Textura franco-arcillo-arenosa: $48 \%-75 \%$ arena y $20 \%-35 \%$ arcilla.

**Textura franco-arenosa: $52 \%-70 \%$ arena $y<20 \%$ arcilla.

Tabla 3. Distribución del fertilizante requerido de acuerdo con los tratamientos evaluados.

\begin{tabular}{|c|c|c|c|c|c|c|c|c|c|c|c|c|c|}
\hline \multirow{2}{*}{ Tratamiento } & \multirow{2}{*}{$\frac{\text { Frecuencia }}{N^{\circ} \text { aplicaciones/año }}$} & \multicolumn{12}{|c|}{ Mes de aplicación } \\
\hline & & Ene & Feb & Mar & Abr & May & Jun & Jul & Ago & Sep & Oct & Nov & Dic \\
\hline 1 & 2 & & & & & & & & & & & & \\
\hline 2 & 3 & & & & & & & & & & & & \\
\hline 3 & 4 & & & & & & & & & & & & \\
\hline 4 & 6 & & & & & & & & & & & & \\
\hline
\end{tabular}

Los fertilizantes utilizados correspondieron a fuentes simples o mezcla de las mismas realizadas en cada localidad, según lo sugerido por el análisis de suelos, utilizando para tal fin urea, fosfato diamónico-DAP, cloruro de potasio y/o kieserita y óxido de magnesio; para suplir los requerimientos de nitrógeno, fósforo, potasio y magnesio, respectivamente.

El análisis de la información se basó en los siguientes criterios: para cada localidad y tratamiento se estimó el promedio y la variación de la variable de respuesta producción de café cereza (cc), durante los pases de recolección y se consolidó la producción de cada año y la producción total, correspondiente al final de los cuatro años de cosecha.

Posteriormente, se hizo un análisis de varianza asociado al diseño experimental bloques completos al azar, con siete repeticiones, con la variable de respuesta (producción de café cereza) al 5\%. Cuando el análisis de varianza mostró efecto de los tratamientos, se aplicó la prueba de Tukey al $5 \%$ para determinar los tratamientos con mayor producción con respecto al testigo relativo (tratamiento 1). 
Con base en la información obtenida a partir de la Plataforma Agroclimática de Cenicafé (agroclima.cenicafe.org), se obtuvo la pluviosidad mensual durante la vigencia del experimento en cada localidad, con el fin de establecer de manera descriptiva las tendencias en el incremento o disminución de la lluvia frente a un mínimo histórico de 30 años según la estación agroclimática.

\section{RESULTADOS}

La producción de café cereza presentó variaciones entre las localidades como consecuencia de las diferentes condiciones agroecológicas tales como horas luz, horas de brillo solar, lluvia y temperatura; los cuales determinan el crecimiento, el desarrollo y la producción del café en Colombia (FNC, 2013).

\section{Variación de la producción durante los años del estudio}

En general, el primer año de producción registró un bajo nivel de cosecha por tratarse de plantaciones que se encontraban en período de transición entre la fase de crecimiento vegetativo y la reproductiva. Dicho fenómeno no fue evidente en El Agrado, en vista de que la plantación presentaba 24 meses de edad al momento del inicio de la aplicación de los tratamientos, esto significa que una diferencia de seis meses pudo haber permitido el desarrollo de una mayor cantidad de biomasa vegetativa (ramas y nudos principalmente), en la cual puede albergarse una mayor cantidad de flores y frutos, respecto a un cultivo de menor edad (18 meses), como el de las otras localidades evaluadas.

Con base en las anteriores consideraciones, los resultados presentaron la tendencia que se describe a continuación:

\section{Producción anual}

Mientras que, en El Agrado, El Rosario, Naranjal, El Porvenir y San Antonio, no se presentó efecto del fraccionamiento de la fertilización en la producción anual de café cereza durante la vigencia del experimento (Figuras 1, 2, 3, 5 y 6), en Paraguaicito la aplicación del fertilizante fraccionado cuatro y seis veces por año, condujo a incrementar la producción de café entre un $10 \%$ y $12 \%$ respecto a los demás tratamientos evaluados (P>0,01) durante 2013 (Figura 4).

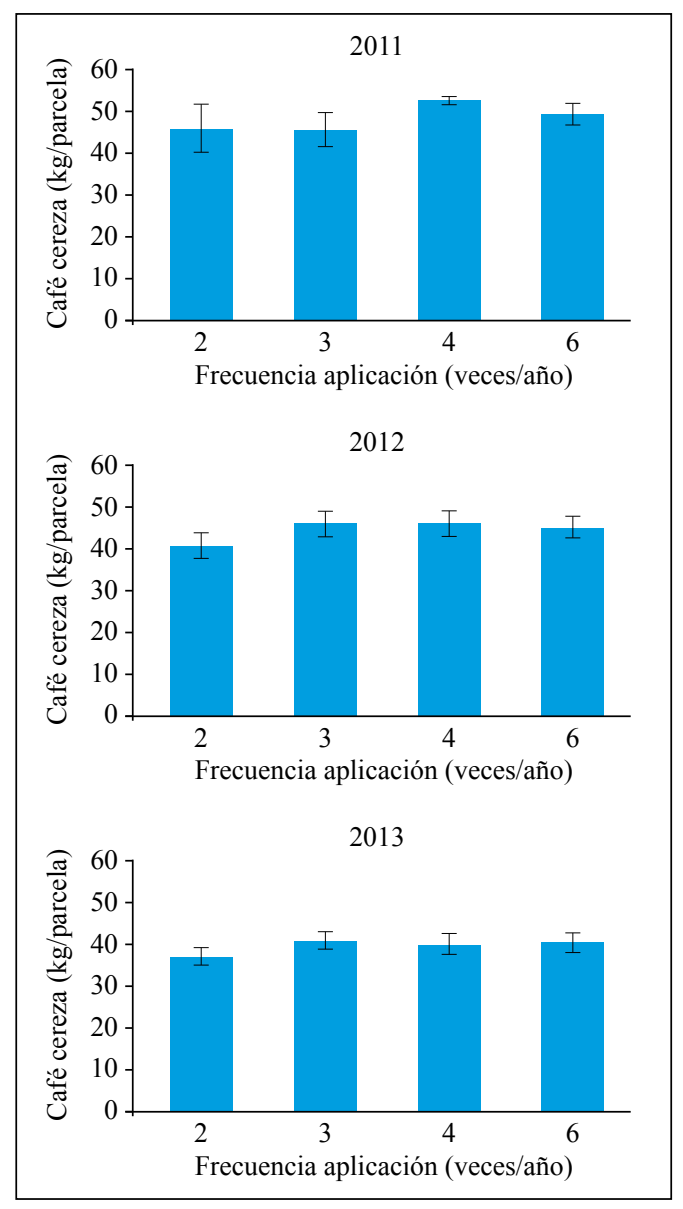

Figura 1. Promedios y variación (error estándar) de la producción anual de café cereza en El Agrado. 


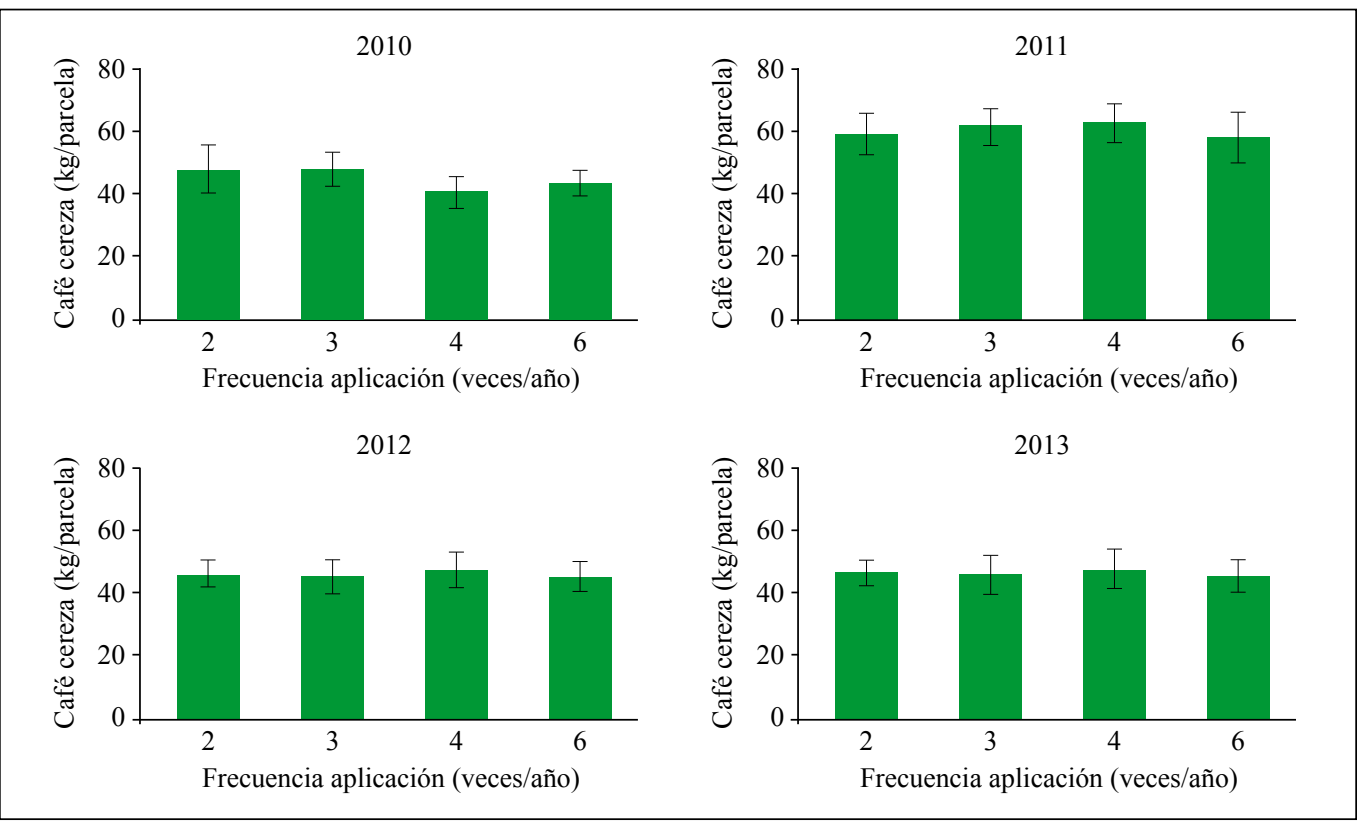

Figura 2. Promedios y variación (error estándar) de la producción anual de café cereza en El Rosario, por efecto del fraccionamiento de la fertilización.

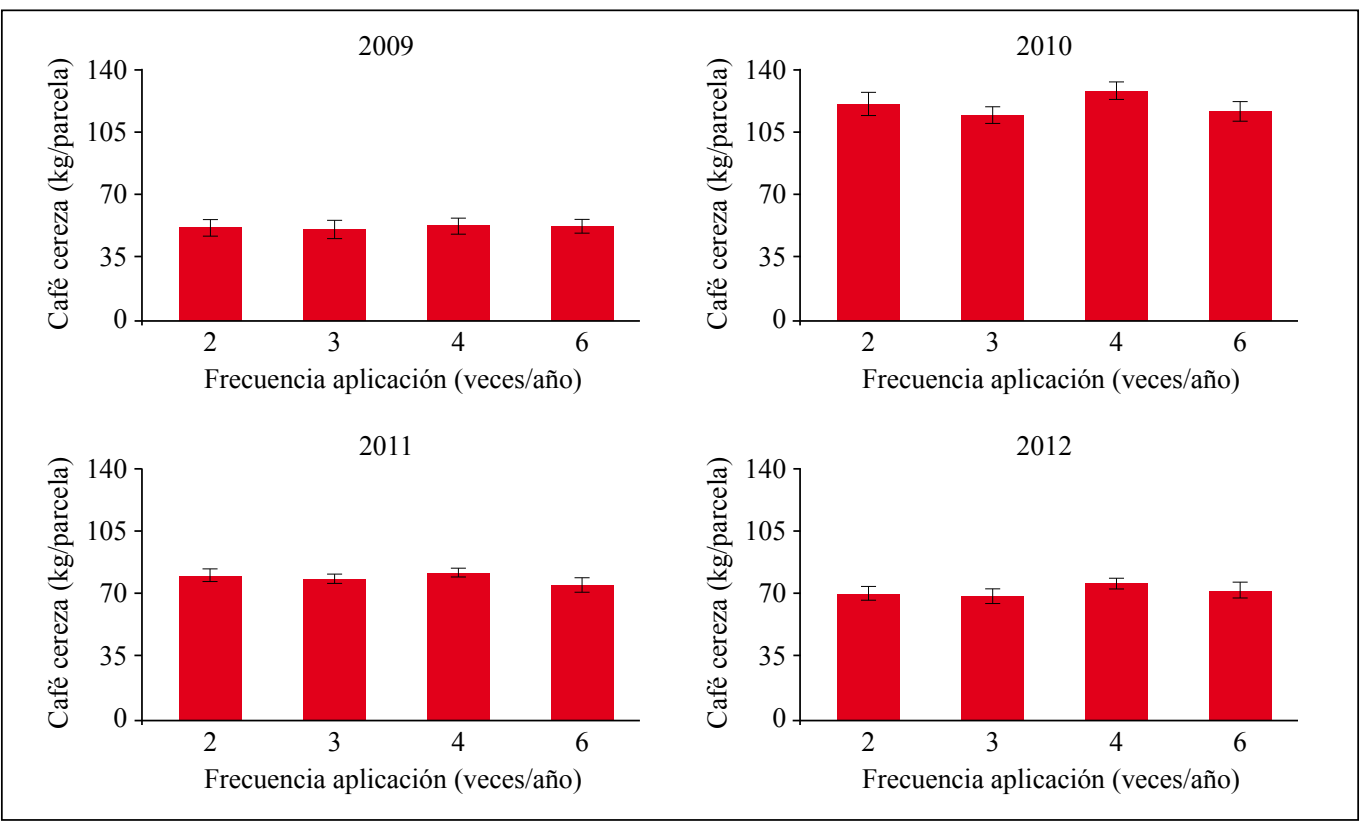

Figura 3. Promedios y variación (error estándar) de la producción anual de café cereza en Naranjal. 


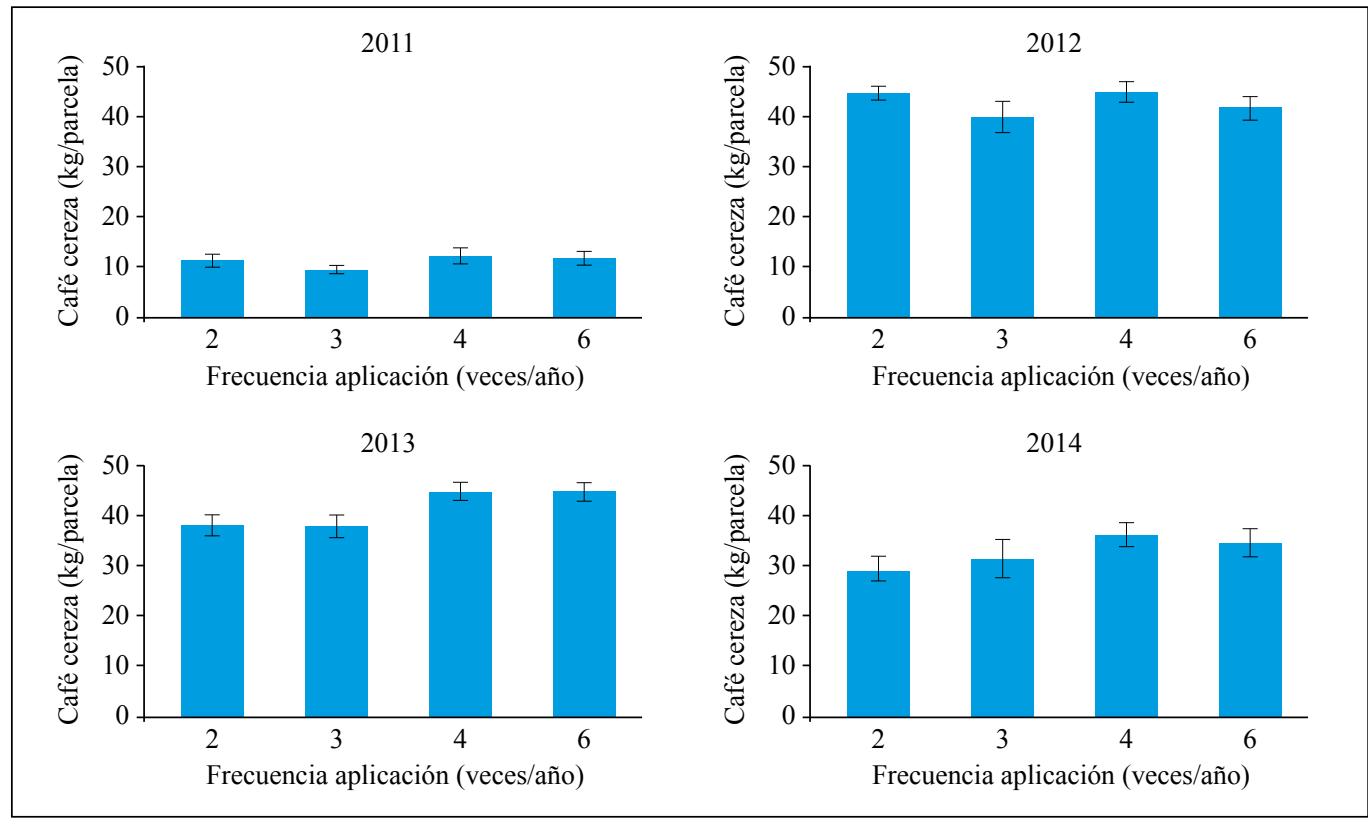

Figura 4. Promedios y variación (error estándar) de la producción anual de café cereza en Paraguaicito.

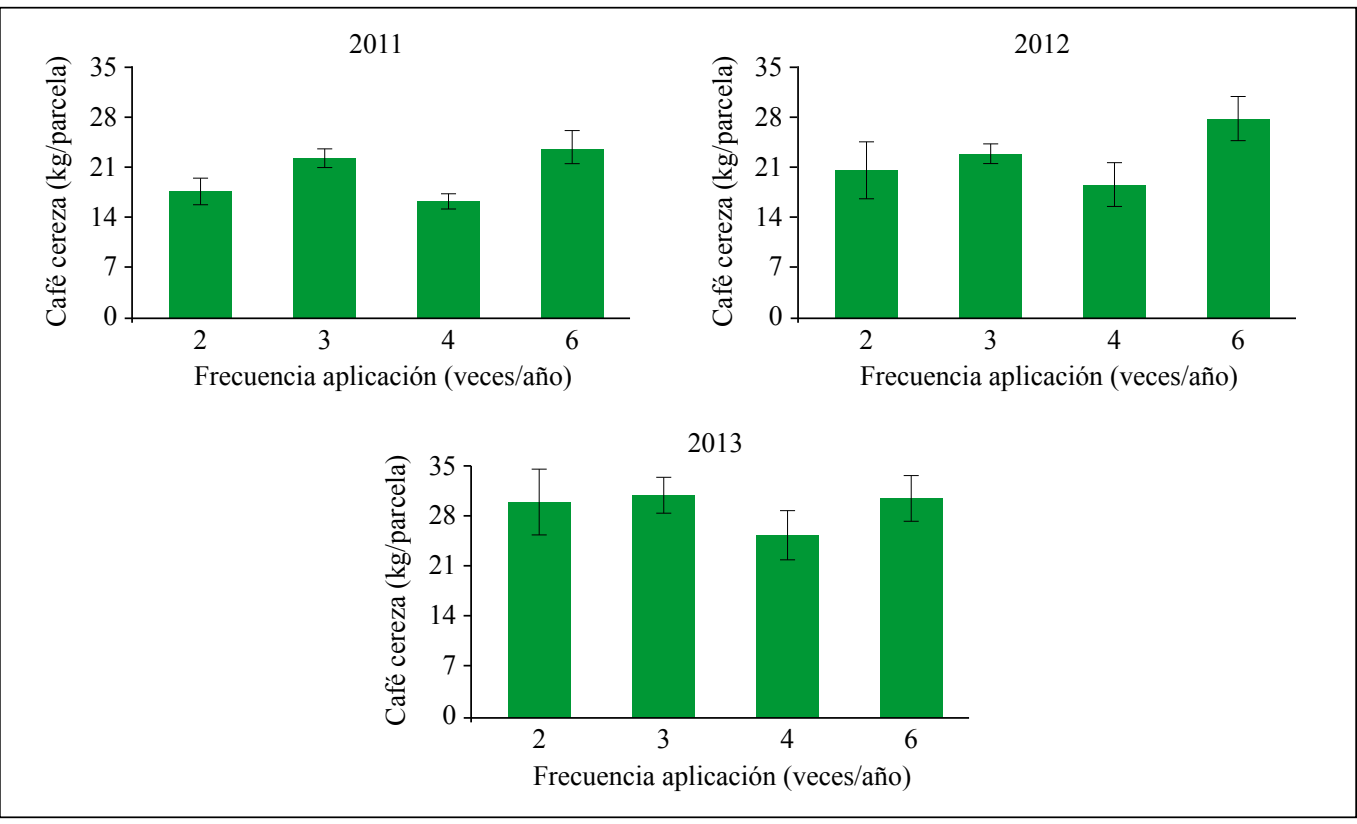

Figura 5. Promedios y variación (error estándar) de la producción anual de café cereza en San Antonio. 


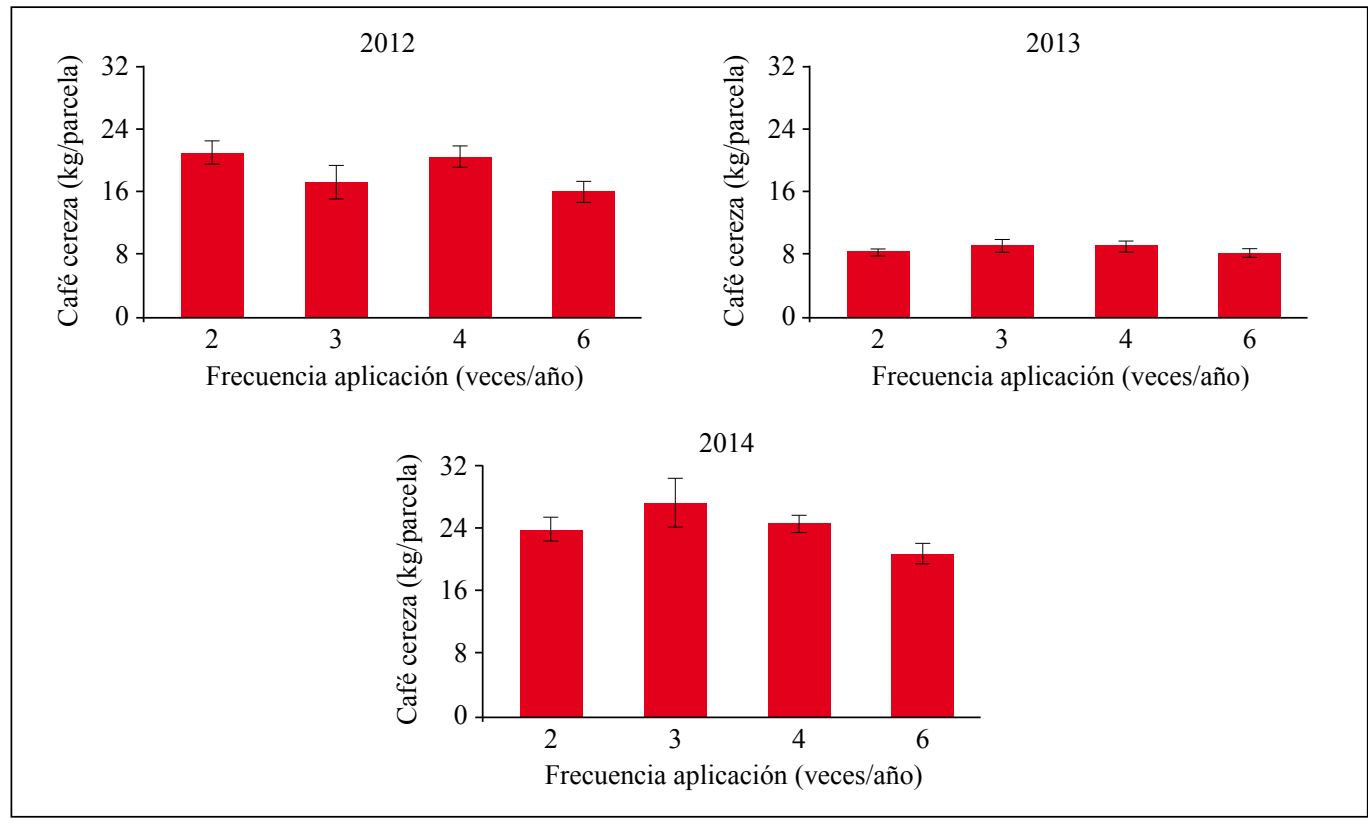

Figura 6. Producción promedio anual de café cereza y acumulado de cosecha 2012-2014 en El Porvenir.

\section{Producción acumulada}

Con relación al acumulado de la producción, durante los diferentes años en los que se condujo el experimento para cada localidad objeto de estudio (Tabla 4), la práctica de fraccionar el fertilizante requerido cuatro y seis veces por año durante el último año de cosecha, influyó significativamente $(\mathrm{P}>0,02)$ sólo en Paraguaicito, en el acumulado del ciclo de producción (cuatro años). Para los demás sitios donde se desarrolló el estudio, no hubo efecto del fraccionamiento de la fertilización. Esto indica que la cantidad total de fertilizante por año puede suministrarse en dos o tres aplicaciones, por ser esta una labor que demanda menor cantidad de mano de obra en su implementación.

\section{Tendencias de la lluvia en las diferentes áreas experimentales}

La cantidad de lluvia registrada en las diferentes áreas donde se llevó a cabo la investigación estuvo durante al menos dos de los años evaluados, por encima de los registros históricos (Figura 7). Respecto a su distribución, esta fue de tipo bimodal en cinco de las localidades, distinguiéndose a nivel general marzo-abril y septiembreoctubre los dos períodos que en el año se presentaron las mayores precipitaciones $\mathrm{y}$, por ende, fisiológicamente ha sido demostrado, constituyen dos momentos que marcan la fenología del cultivo. En El Porvenir, donde históricamente las precipitaciones superan los $4.000 \mathrm{~mm}$ año ${ }^{-1}$, y en San Antonio con lluvia histórica inferior a todos los demás sitios, no se presentó el patrón de distribución antes mencionado.

Considerando lo anterior y en aras de tener mayores elementos para la discusión de los resultados, se presentan los registros de precipitación de los 12 meses que antecedieron al año de inicio de la aplicación de los tratamientos. 
Tabla 4. Promedio de producción acumulada de café cereza por localidad. Letras no comunes indican diferencia estadística entre tratamientos (frecuencia de aplicación) según prueba Tukey al 5\%.

\begin{tabular}{|c|c|c|c|c|c|c|c|c|c|}
\hline \multirow{4}{*}{$\begin{array}{c}\begin{array}{c}\text { Frecuencia aplicación } \\
\text { (No aplicaciones/año) }\end{array} \\
2\end{array}$} & \multicolumn{9}{|c|}{ Acumulado producción (kg café cereza/parcela) } \\
\hline & \multicolumn{3}{|c|}{ El Agrado } & \multicolumn{3}{|c|}{ EI Rosario } & \multicolumn{3}{|c|}{ El Porvenir } \\
\hline & \multicolumn{2}{|c|}{ Promedio } & \multirow{2}{*}{$\frac{\text { CV }}{22,43}$} & \multicolumn{2}{|c|}{ Promedio } & \multirow{2}{*}{$\frac{\mathbf{C V}}{25,33}$} & \multicolumn{2}{|c|}{ Promedio } & \multirow{2}{*}{$\begin{array}{c}\text { CV } \\
14,60\end{array}$} \\
\hline & 123,00 & $\mathrm{a}$ & & 212,70 & $\mathrm{a}$ & & 53,56 & a & \\
\hline 3 & 132,10 & $\mathrm{a}$ & 16,10 & 218,10 & a & 24,52 & 53,96 & a & 16,52 \\
\hline 4 & 138,20 & a & 9,52 & 215,50 & $\mathrm{a}$ & 24,18 & 54,44 & a & 9,43 \\
\hline \multirow[t]{3}{*}{6} & 134,20 & $\mathrm{a}$ & 6,95 & 209,24 & $\mathrm{a}$ & 24,91 & 45,40 & $\mathrm{a}$ & 14,90 \\
\hline & \multicolumn{3}{|c|}{ San Antonio } & \multicolumn{3}{|c|}{ Naranjal } & \multicolumn{3}{|c|}{ Paraguiacito } \\
\hline & \multicolumn{2}{|c|}{ Promedio } & $\mathrm{CV}$ & \multicolumn{2}{|c|}{ Promedio } & $\mathrm{CV}$ & \multicolumn{2}{|c|}{ Promedio } & $\mathrm{CV}$ \\
\hline 2 & 81,03 & $\mathrm{a}$ & 29,81 & 324,91 & $\mathrm{a}$ & 13,53 & 121,69 & $\mathrm{~b}$ & 12,94 \\
\hline 3 & 85,33 & $\mathrm{a}$ & 11,53 & 313,77 & $\mathrm{a}$ & 12,04 & 116,63 & $\mathrm{~b}$ & 19,29 \\
\hline 4 & 69,84 & $\mathrm{a}$ & 26,77 & 339,97 & $\mathrm{a}$ & 7,32 & 136,75 & $\mathrm{a}$ & 11,80 \\
\hline 6 & 94,90 & $\mathrm{a}$ & 23,68 & 318,09 & $\mathrm{a}$ & 13,28 & 131,38 & $\mathrm{a}$ & 14,08 \\
\hline
\end{tabular}

\section{DISCUSIÓN}

Los resultados demuestran que, para cinco de las seis localidades de la zona cafetera de Colombia en la que se llevó a cabo el estudio, fraccionar más de dos veces por año la cantidad de fertilizante requerida para cafetales en edad productiva según el análisis de suelos, no condujo a incrementar la producción.

Esto indica que la eficiencia de la fertilización en el cultivo de café involucra la acción de factores relacionados con el suelo y la selección de fuentes fertilizantes que, aplicados bajo condiciones climáticas específicas, conducen a suplir adecuada y oportunamente los requerimientos nutricionales necesarios para la producción del grano, sin comprometer el desarrollo de estructuras que soportan las cosechas siguientes.
En concordancia con lo anterior, en un ámbito general y desde el punto de vista climático, durante los años en los que se llevó a cabo la investigación fue evidente un incremento en las lluvias respecto al histórico, particularmente en aquellas localidades donde se hicieron evaluaciones entre 2009 y 2011, período que de acuerdo con el Ideam (2011), se presentó un evento climático La Niña catalogado como el más intenso de las últimas cuatro décadas. Por lo tanto, la pluviosidad analizada como único factor responsable de las pérdidas de nutrientes en el agua de percolación, tuvo que haber generado un impacto negativo en la producción de café cuando el fertilizante suministrado se fraccionó dos veces en el año (tratamiento 1), dado que a un mayor nivel de lluvia corresponderían incrementos en la pérdida de nutrientes por lixiviación $y$, por consiguiente, menor cantidad de elementos 


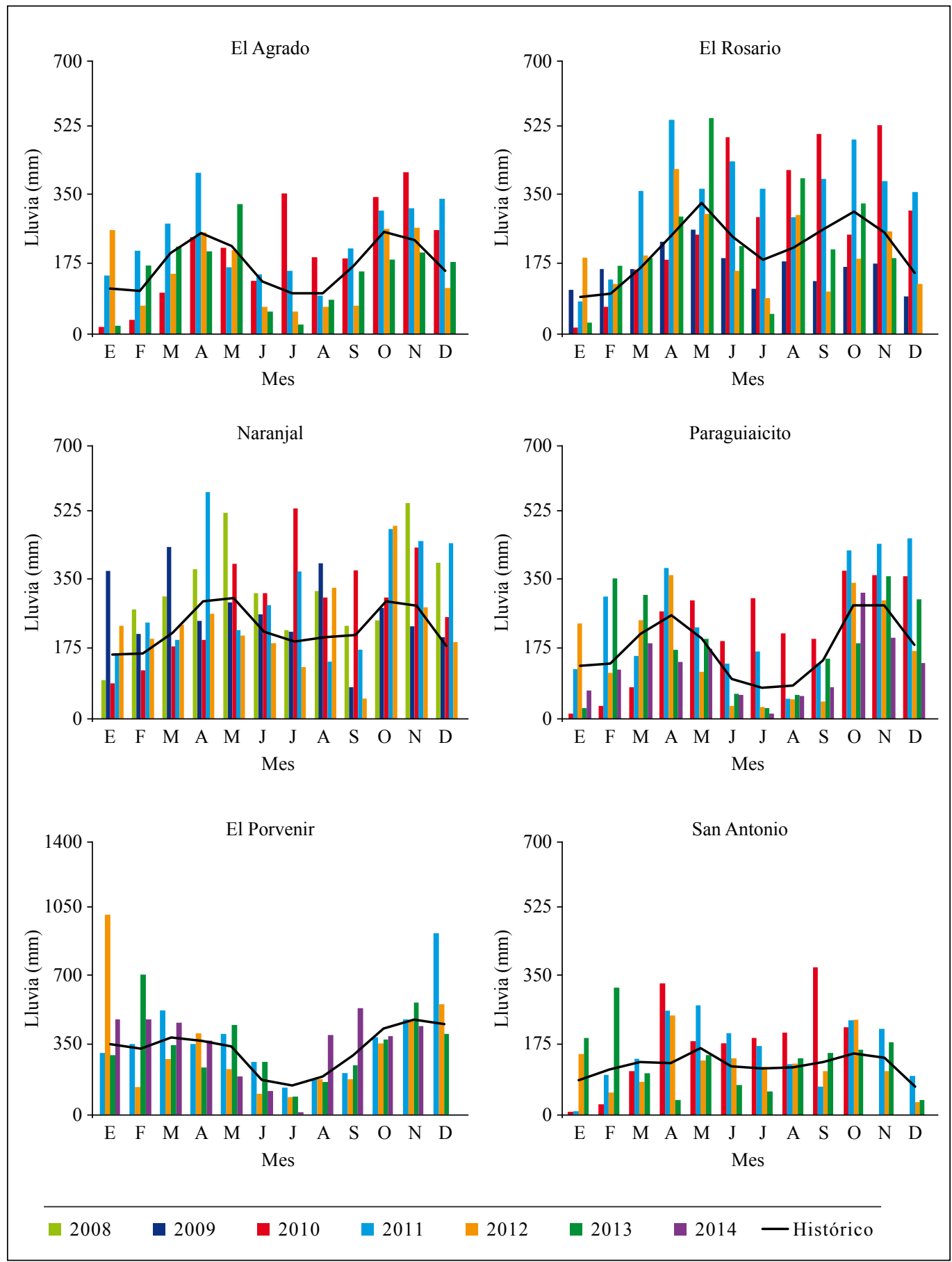

Figura 7. Lluvia mensual e histórica para las localidades donde se desarrolló la investigación. 
minerales para que la planta alcance su potencial de crecimiento y producción.

Es necesario entonces sumar a las anteriores circunstancias el tipo de suelo, dado que, dependiendo de sus características químicas y físicas, la pluviosidad puede inducir a que los nutrientes aplicados vía fertilización sean aprovechados por las plantas o escapen del sistema productivo en el corto, mediano o largo plazo (Georgiadis et al., 2017). Desde el punto de vista de las características químicas, resulta evidente que se proporcionaron los nutrientes y enmiendas sugeridas según los resultados del análisis de suelos realizado cada dos años, como lo recomienda Sadeghian (2008), aspecto que ratifica una adecuada periodicidad en el seguimiento a las condiciones nutricionales del suelo para la reposición de elementos extraídos y removidos por la planta, al tiempo de realizar los respectivos ajustes al plan de fertilizaciones y acondicionamientos en caso de haber sido necesario. Por otro lado, desde el punto de vista físico, se presume que la textura, especialmente aquella con predominio de arenas, condiciona la permanencia de los nutrientes aplicados vía fertilización ( $\mathrm{Lu}$ et al., 2019) aunque dicho efecto puede depender del nutriente y la fuente empleada (Arias y Sadeghian, 2018). Sin embargo, es oportuno anotar que, en las condiciones del estudio, los lotes presentaron dos clases texturales, franco-arcillo-arenosa y franco-arenosa, en las cuales el contenido de arenas puede estar entre el $48 \%$ y el $75 \%$. Aun así, los resultados en producción por efecto de la fertilización fraccionada tuvieron un comportamiento similar en las diferentes áreas del estudio.

Los resultados obtenidos entran en concordancia con lo encontrado por Uribe y Mestre (1980), salvo que, para el caso de estos autores, las cantidades de fertilizante objeto de estudio fueron superiores a lo actualmente recomendado. En consecuencia, la residualidad de las fuentes objeto de estudio por efecto de la alta cantidad, pudo haber sido uno de los aspectos que condujo a enmascarar el posible efecto de su fraccionamiento a través del tiempo. Aun así, en una de las localidades evaluadas cuya pluviosidad superó $3.200 \mathrm{~mm}$ año-1, encontraron efecto del fraccionamiento durante uno de los años del estudio, particularmente cuando se utilizó la dosis más baja de fertilizante.

En Paraguaicito, sitio donde el fraccionamiento anual de la fertilización en cuatro y seis aplicaciones condujo al aumento del $12 \%$ en la producción para dos años evaluados y el acumulado del ciclo de producción, dicha respuesta puede tener explicación al asociar las condiciones climáticas con las características del suelo. Es decir, la conductividad hidráulica característica de este suelo, la cual según González y Sadeghian (2012), supera en un $70 \%$ a la de Naranjal (sitio donde la respuesta en producción fue independiente del fraccionamiento de la fertilización) puede facilitar la pérdida de nutrientes, particularmente en momentos en los cuales la planta puede necesitarlos más. Hansen y Eriksenm (2016) sostienen que la lixiviación de nitrógeno, nutriente más determinante para la producción de café en Colombia, puede ser significativamente alta en suelos en cuya fase sólida predominan las arenas gruesas, especialmente en épocas de alta precipitación.

Lo mencionado sugiere que la incidencia de la alta precipitación sobre una región cuyas características de suelo favorecen una rápida infiltración del agua que llega al suelo y, por ende, una mayor lixiviación de los nutrientes disueltos en ella, pueden brindar pautas para tomar la decisión acerca de las bondades que puede tener el fraccionamiento de la fertilización en la producción de café. Sustento de ello es que, durante 2013 la pluviosidad registrada 
entre marzo y abril, época en la que coincide la aplicación de todos los tratamientos (Tabla 3), la lluvia registrada superó entre 30\% y $60 \%$ al histórico de 50 años de observaciones (Figura 7), lo cual pudo inducir a la lixiviación de nutrientes en la totalidad de tratamientos; aunque la magnitud del fenómeno sería diferente tratándose de uno u otro. Por ejemplo, en los tratamientos 1 y 2 que son portadores del 50\% y $33 \%$ del fertilizante total requerido por año, respectivamente, habría un mayor impacto si se comparan con los tratamientos 3 y 4 , en cuyo caso el evento climático pondría en vulnerabilidad el 25\% (tratamiento 3 ) o el $17 \%$ (tratamiento 4) del total del fertilizante suministrado. Sadeghian et al., (2013), sostienen que los dos meses luego de la floración, la cual es evidente en Paraguiacito entre enero y febrero, se presenta la mayor acumulación de nutrientes en los frutos de café que llegan a su madurez de recolección seis meses después, lo cual para este caso corresponde al período comprendido entre septiembre y octubre del mismo año.

Lo anterior, puede explicar la respuesta al fraccionamiento, tanto en 2013 como para 2014. Circunstancia que puede asociarse con los aspectos que condicionan los tiempos fenológicos de un cultivo perenne como el café. Arcila (2007) concluye que, a partir de sus investigaciones, para las condiciones de la zona cafetera colombiana son necesarios 18 meses para la formación de una cosecha, es decir, se requieren entre cuatro y seis meses para generar ramas y nudos que serán soporte de unas estructuras florales, que necesitan de un período entre tres y cuatro meses para su total desarrollo y, a partir de la apertura floral, se requieren ocho meses adicionales para que el fruto llegue al estado de madurez para la recolección. Esto quiere decir que, cualquiera que fuese el plan de fraccionamiento de fertilización, este tuvo que haber impactado con mayor intensidad el año inmediatamente anterior, dado que, para esta época, las estructuras de la planta que soportaron la producción, son en buena medida las que se empezaron a formar desde el inicio de 2013.

Para los sitios donde los resultados de este estudio han demostrado que fraccionar en más de dos oportunidades el fertilizante requerido según el análisis de suelos no afecta la producción, puede considerarse esta práctica como una opción para aquellos caficultores que no cuentan con los recursos para la adquirir la totalidad del fertilizante recomendado por año o por semestre, siempre y cuando se consideren las tendencias que sobre la variación climática divulguen las instituciones. Esto significa que mientras en un año con fenómeno de La Niña la fertilización podría llevarse a cabo fraccionada dos, tres, cuatro o seis veces tal como lo demuestra este estudio, durante un año con tendencia a la ocurrencia de un evento El Niño, debería tenerse la precaución de programar esta práctica en sólo dos oportunidades, pues se corre el riesgo de que la fertilización requerida por año quede incompleta como lo expresan González et al., (2014), dado que puede no haber certeza de la época del retorno de las lluvias, insumo que gobierna la humedad del suelo y brinda la posibilidad para que los nutrientes contenidos en los fertilizantes pasen a formar parte de la reserva del suelo y/o sean adquiridos por el cultivo.

Por último, es bien sabido que el tipo de fertilizantes y su forma de aplicación contribuyen significativamente con la eficiencia de la nutrición, específicamente cuando se utilizan fuentes como la urea, cuya reacción en el suelo conduce a que parte del nitrógeno que contiene se pierda por volatilización o se lixivie (González y Sadeghian, 2012a, 2012b). Pese a esto, estudios donde se han evaluado las citadas pérdidas han demostrado que para el caso de la volatilización en el cultivo del 
café, esta puede disminuir cuando la urea se aplica esparcida sobre una superficie de suelo húmedo (González et al., 2015); en tanto que el potencial de lixiviación puede variar en función de que las condiciones edáficas favorezcan a la conversión preferencial de formas inorgánicas como los nitratos o amonio (Sadeghian et al., 2015), aspectos que pueden ser altamente variables en el tiempo y en el espacio, aun en cortas distancias.

Los demás nutrimentos aplicados en los planes de fertilización del estudio, teóricamente presentarían sólo susceptibilidad a perderse por lixiviación o a fijarse en la matriz del suelo. Bajo este esquema, la investigación se ha centrado en el ámbito académico e industrial en proponer estrategias tendientes a la eficiencia en la fertilización, entre las que se destacan el uso de fertilizantes con recubrimiento (Niemiec et al., 2015; Tian et al., 2018), el manejo de la acidez del suelo (Maresca et al., 2019) y el fraccionamiento de la fertilización. Todas de manera conjunta o adoptadas individualmente con resultados alentadores en cultivos de ciclo corto y en áreas con estacionalidad en el clima (Sowinski y Galb, 2018; Liu et al., 2017) o para nutrientes específicos como es el caso de nitrógeno en café en Brasil (Meriguetti et al., 2017), pero con respuesta no del todo satisfactoria en programas integrales de nutrición para el café y para las regiones cafeteras diversas en sus recursos climáticos y edafológicos, como lo demuestra esta investigación.

En el presente estudio se utilizaron fertilizantes de uso común en la caficultura del país, urea, DAP, $\mathrm{KCl}$ y kieserita u óxido de magnesio, como fuentes de nitrógeno, fósforo, potasio y magnesio, respectivamente. Todos se suministraron en mezcla física, esparcidos sobre la superficie del suelo, alrededor de la zona de raíces de los árboles y sin cubrir, por ser esta una alternativa eficaz y de bajo costo, comparada con la aplicación localizada y cubierta con el suelo, según lo demostraron Mestre y Uribe (1980). Dicha modalidad de aplicación según lo discutido anteriormente, implica que los fenómenos naturales que determinan las pérdidas de los componentes incluidos en las fuentes fertilizantes hayan actuado sin restricción. Este aspecto sustenta que los resultados obtenidos pueden estar satisfactoriamente explicados en función de las variables climáticas, de suelos y de fisiología de las plantas anteriormente discutidas.

\section{LITERATURA CITADA}

Afshar, R., Lin, R., Mohammed, Y. A., \& Chen, C. (2018). Agronomic effects of urease and nitrification inhibitors on ammonia volatilization and nitrogen utilization in a dryland farming system: Field and laboratory investigation. Journal of Cleaner Production, 172, 4130 - 4139. https://doi.org/10.1016/j.jclepro.2017.01.105

Arcila, J. (2007). Crecimiento y desarrollo de la planta de café. En J. Arcila, F. Farfán, A. Moreno, L, Salazar, \& E. Hincapie (Eds.), Sistemas de producción de café en Colombia: crecimiento, desarrollo, floración y producción. (1ra ed., pp. 21-60). Cenicafé. http://hdl. handle.net/10778/720

Arias-Suarez, E., \& Sadeghian, S. (2018). Lixiviación de potasio en suelos de la zona cafetera y su relación con la textura. Biotecnología en el sector agropecuario y agroindustrial. 16 (1). 34-42. https:// doi.org/10.18684/10.18684/bsaa.v16n1.632

Federación Nacional de Cafeteros de Colombia. (2013). Manual del cafetero colombiano: Investigación y tecnología para la sostenibilidad de la caficultura (Vol. 3). Cenicafé.

Ghiberto, P. J., Libardi, P. L., Brito, A. S., \& Trivelin, P. C. O. (2009). Leaching of nutrients from a sugarcane crop growing on an Ultisol in Brazil. Agricultural Water Management, 96(10), 1443-1448. https:/doi. org/10.1016/j.agwat.2009.04.020

Georgiadis, P., Taeroe, A., Stupak, I., Kepfer-Rojas, S., Zhang, W.,Pinheiro-Bastos, R., \& Raulund-Rasmussen, K. (2017). Fertilization effects on biomass production, nutrient leaching and budgets in four stand development stages of short rotation forest poplar. Forest Ecology 
and Management, 397, 18-26. https://doi.org/10.1016/j. foreco.2017.04.020

González, H., \& Sadeghian, S. (2012). Lixiviación de nitrógeno en suelos de la zona cafetera a partir de diferentes fuentes fertilizantes. Revista Cenicafé, 63(1), 111-119. http://hdl.handle.net/10778/516

González, H., Sadeghian, S., \& Jaramillo, A. (2014). Épocas recomendables para la fertilización de cafetales. Avances Técnicos Cenicafé, 442, 1-12.http://hdl.handle. net/10778/498

González, H., Sadeghian, S., Castro, A. F., \& Medina, R. D. (2015). Alternativas para disminuir la volatilización de nitrógeno producida por la fertilización con urea. Revista Cenicafé, 66(1), 7-16. http://hdl.handle.net/10778/604

Hansen, E. M., \& Eriksen, J. (2016). Nitrate leaching in maize after cultivation of differently managed grassclover leys on coarse sand in Denmark. Agriculture, Ecosystems and Environment, 216, 309-313. https:// doi.org/10.1016/j.agee.2015.10.010

Ierna, A., \& Mauromicale, G. (2018). Potato growth, yield and water productivity response to different irrigation and fertilization regimes. Agricultural Water Management, 201, 21-26. https://doi.org/10.1016/j. agwat.2018.01.008

Instituto de Hidrología, Meteorología y estudios Ambientales - IDEAM. (2011). El fenómeno de "La Niña" presenta su etapa de madurez; algunos modelos y análisis realizados por el IDEAM indican una Niña fuerte. Boletín Informativo, 22. 1-7.

Liu, S., Wang, J. J., Tian, Z., Wang, X., \& Harrison, S. (2017). Ammonia and greenhouse gas emissions from a subtropical wheat field under different nitrogen fertilization strategies. Journal of Environmental Sciences, 57, 196-210. https://doi.org/10.1016/j. jes.2017.02.014

Lu, J., Bai, Z., Velthof, G.L., Wu, Z., Chadwick, D., \& Ma, L. (2019). Accumulation and leaching of nitrate in soils in wheat-maize production in China. Agricultural Water Management, 212, 407-415. https://doi.org/10.1016/j. agwat.2018.08.039

Maresca, A., Krüger, O., Herzel, H., Adam, C., Kalbe, U., \& Astrup, T. F. (2019). Influence of wood ash pretreatment on leaching behaviour, liming and fertilising potential. Waste Management, 83, 113-122. https://doi. org/10.1016/j.wasman.2018.11.003

McArthur, J. W., \& McCord, G. C. (2017). Fertilizing growth: Agricultural inputs and their effects in economic development. Journal of Development Economics, 127, 133-152.https://doi.org/10.1016/j.jdeveco.2017.02.007
Merigueti, V., Pereira, I., Lier, Q., Dourado-Neto, D., \& Reichard, K. (2017). Environmental benefits of reducing $\mathrm{N}$ rates for coffee in the Cerrado. Soil \& Tillage Research, 166, 76-83. https://doi.org/10.1016/j.still.2016.10.006

Mestre, A., \& Uribe, A. (1980). Dosis y frecuencia de aplicación del fertilizante en la producción de café. Revista Cenicafé, 31(4), 145-163. http://hdl.handle. net/10778/684

Niemiec, M., Cupiał, M., \& Szeląg-Sikora, A. (2015). Efficiency of celeriac fertilization with phosphorus and potassium under conditions of integrated plant production. Agriculture and Agricultural Science Procedia, 7, 184-191. https://doi.org/10.1016/j. aaspro.2015.12.015

Sadeghian, S., Hernández, E., \& González, H.(2007). Mezcla de fertilizantes en la finca, una buena opción para el caficultor. Avances Técnicos Cenicafé, 362, 1-8. http:// hdl.handle.net/10778/363

Sadeghian, S. (2008). Fertilidad del suelo y nutrición del café en Colombia: Guía Práctica. Boletín Técnico Cenicafé, 32, 1-43. http://hdl.handle.net/10778/587

Sadeghian, S., \& González, H. (2012). Alternativas generales de fertilización para cafetales en la etapa de producción. Avances Técnicos Cenicafé, 424, 1-8. http:// hdl.handle.net/10778/1107

Sadeghian, S., Mejía, B., \& González,H.(2013).Acumulación de calcio magnesio y azufre en los frutos de café Avances Técnicos Cenicafé, 430, 1-8. http://hdl.handle. net $/ 10778 / 408$

Sadeghian, S., González, H., \& Arias, E. (2015). Lixiviación de nutrientes en suelos de la zona cafetera: Prácticas que ayudan a reducirla. Boletín Técnico Cenicafé, 40, 1-34. http://hdl.handle.net/10778/1110

Sowiński, J., \& Głąb, L. (2018). The effect of nitrogen fertilization management on yield and nitrate contents in sorghum biomass and bagasse. Field Crops Research, 227, 132-143.https://doi.org/10.1016/j.fcr.2018.08.006

Tian, X., Li, C., Zhang, M., Li, T., Lu, Y., \& Liu, L. (2018). Controlled release urea improved crop yields and mitigated nitrate leaching under cotton-garlic intercropping system in a 4-year field trial. Soil and Tillage Research, 175, 158-167. https://doi. org/10.1016/j.still.2017.08.015

Tully, K. L., Lawrence, D., \& Scanlon, T. M. (2012). More trees less loss: Nitrogen leaching losses decrease with increasing biomass in coffee agroforests. Agriculture, Ecosystems \& Environment, 161, 137-144. https://doi. org/10.1016/j.agee.2012.08.002 